\title{
Inhibition of airway remodeling in IL-5-deficient mice
}

\section{Jae Youn Cho, Marina Miller, Kwang Je Baek, Ji Won Han, Jyothi Nayar, Sook Young Lee, Kirsti McElwain, Shauna McElwain, Stephanie Friedman, and David H. Broide}

Department of Medicine, University of California San Diego, La Jolla, California, USA

\begin{abstract}
To determine the role of IL-5 in airway remodeling, IL-5-deficient and WT mice were sensitized to OVA and challenged by repetitive administration of OVA for 3 months. IL-5-deficient mice had significantly less peribronchial fibrosis (total lung collagen content, peribronchial collagens III and V) and significantly less peribronchial smooth muscle (thickness of peribronchial smooth muscle layer, $\alpha$-smooth muscle actin immunostaining) compared with WT mice challenged with OVA. WT mice had a significant increase in the number of peribronchial cells staining positive for major basic protein and TGF- $\beta$. In contrast, IL-5-deficient mice had a significant reduction in the number of peribronchial cells staining positive for major basic protein, which was paralleled by a similar reduction in the number of cells staining positive for TGF- $\beta$, suggesting that eosinophils are a significant source of TGF- $\beta$ in the remodeled airway. OVA challenge induced significantly higher levels of airway epithelial $\alpha_{V} \beta_{6}$ integrin expression, as well as significantly higher levels of bioactive lung TGF- $\beta$ in WT compared with IL-5-deficient mice. Increased airway epithelial expression of $\alpha_{V} \beta_{6}$ integrin may contribute to the increased activation of latent TGF- $\beta$. These results suggest an important role for IL-5, eosinophils, $\alpha_{\mathrm{V}} \beta_{6}$, and TGF- $\beta$ in airway remodeling.
\end{abstract}

J. Clin. Invest. 113:551-560 (2004). doi:10.1172/JCI200419133.

\section{Introduction}

The accumulation of peribronchial eosinophils is a prominent feature of airway inflammation in asthma (1). Several cytokines and chemokines regulate the trafficking of eosinophils from the bone marrow to the lung (2). One key cytokine regulating the trafficking of eosinophils from the bone marrow to the lung is IL-5, which regulates eosinophil proliferation, differentiation, and release from the bone marrow (1-3). In vivo studies performed in either IL-5-deficient mice (4), IL-5-transgenic mice (5), or mice treated with anti-IL-5 $A b$ 's (6) have suggested an important role for IL- 5 in acute allergen-induced eosinophilic inflammation and airway hyperreactivity. In IL-5-deficient mice there is a significant reduction in the level of bronchoalveolar eosinophila following OVA allergen challenge, which is associated with significantly less airway hyperreactivity to methacholine (4). Similarly, mice treated with an anti-IL-5 Ab prior to allergen challenge have significantly less airway eosinophilia and airway responsiveness $(6,7)$. Not all studies in mice treated with anti-IL-5

Received for publication June 5, 2003, and accepted in revised form November 19, 2003.

Address correspondence to: David Broide, University of California San Diego, Basic Science Building, Room 5090, 9500 Gilman Drive, La Jolla, California 92093-0635, USA. Phone: (858) 534-2374; Fax: (858) 534-2110;

E-mail: dbroide@ucsd.edu.

Conflict of interest: The authors have declared that no conflict of interest exists.

Nonstandard abbreviations used: major basic protein (MBP); bronchoalveolar lavage fluid (BALF); periodic acid Schiff (PAS); latency-associated peptide (LAP).
Ab's have shown inhibition of allergen-induced airway responsiveness, however (8). Transgenic mice that have been molecularly engineered to constitutively express IL-5 in the lung epithelium develop accumulation of peribronchial eosinophils, goblet cell hyperplasia, epithelial hypertrophy, focal collagen deposition, and airway hyperresponsiveness to methacholine in the absence of aerosolized antigen challenge (5). Moreover, adoptive transfer of eosinophils directly into the lungs of OVA-challenged IL-5-deficient mice results in restoration of pulmonary eosinophilia equivalent to that observed in OVA-challenged WT mice, as well as restoration of the development of airway hyperresponsiveness (9). Recent studies suggest that inhibition of both IL-5 and the eosinophil chemoattractant eotaxin results in more complete inhibition of acute allergeninduced eosinophilic inflammation than targeting either IL-5 or eotaxin alone (10). Thus, in the mouse there is considerable evidence that IL-5 plays a prominent role in the development of acute allergen-induced airway eosinophilia, as well as airway responsiveness in most, but not all studies.

In humans with asthma there is also evidence that IL-5 may play a role in the pathogenesis of eosinophilic inflammation and asthma. Airway allergen challenge in asthmatics induces expression of IL-5 by T lymphocytes (11) and eosinophils (12), while increased levels of IL-5 and eosinophil granule proteins can be detected in the airway of symptomatic asthmatics (13). Inhalation of IL-5 induces airway eosinophilia and airway hyperreactivity to methacholine in human asthmatics (14). Initial studies with anti-IL-5 in mild asthmatics demonstrated that anti-IL-5 was effective in inhibiting 
sputum and blood eosinophilia more than $90 \%$ but was not effective in inhibiting the late-phase response to allergen challenge or airway hyperreactivity to methacholine (15). Subsequent studies in asthmatics have demonstrated that while anti-IL-5 is effective in inhibiting blood and sputum eosinophils more than $90 \%$, anti-IL-5 only partially inhibited airway eosinophils (approximately 55\%) and did not reduce the level of airway staining for major basic protein (MBP) (16). Thus, at present, the role of IL-5 and eosinophils in human asthma is unclear.

Asthma is not only characterized by episodes of acute inflammation, but may also be associated with the development of airway remodeling (17). Features of airway remodeling in asthma include the presence of subepithelial fibrosis $(17,18)$ and smooth muscle hypertrophy/hyperplasia $(17,19)$. Because eosinophils express the profibrotic growth factor TGF- $\beta$ (20-23), one of the potential roles of the eosinophil in asthma might include release of TGF- $\beta$ and induction of airway remodeling. Therefore, to determine the role of IL-5 and eosinophils in airway remodeling, as opposed to previous studies investigating its role in acute allergen-induced eosinophilic airway inflammation, we used a recently developed mouse model of airway remodeling to investigate levels of airway remodeling in IL-5-deficient compared with WT mice.

\section{Methods}

\section{Induction of chronic pulmonary eosinophilic} inflammation

IL-5-deficient or littermate WT controls $(n=18-20$ mice/group) were genotyped and used in experiments when they reached 8 weeks of age. The background strain of the WT and IL-5-deficient mice used in this study was C57BL. Mice were immunized intraperitoneally on days 0 and 12 with $50 \mu \mathrm{g}$ of OVA (grade V; Sigma-Aldrich, St. Louis, Missouri, USA) adsorbed to 1 $\mathrm{mg}$ of alum (Sigma-Aldrich) in $200 \mu \mathrm{l}$ normal saline. Intranasal OVA challenges ( $20 \mathrm{ng} / 50 \mu \mathrm{l}$ in PBS) were administered on days 26,29 , and 31 under isoflurane (Vedco Inc., St. Joseph, Missouri, USA) anesthesia and then repeated twice a week for 3 months using a protocol (used in this laboratory) associated with the expression of Th 2 cytokines, including IL-4, IL-5, and IL-9, at 3 months (24). Using this protocol, OVA-sensitized control WT mice repetitively challenged intranasally with PBS do not develop Th2 cytokine expression, eosinophilic inflammation, or airway remodeling changes (assessed by trichrome staining, collagen deposition, smooth muscle thickness, and mucus secretion). Age- and sex-matched control mice were sensitized but not challenged with OVA during the 3-month study. Mice were sacrificed 24 hours after the final OVA challenge, and bronchoalveolar lavage fluid (BALF) and lungs were analyzed. All animal experimental protocols were approved by the University of California, San Diego Animal Subjects Committee.

\section{Processing of lungs for image analysis}

Lungs in the different groups of mice were equivalently inflated with an intratracheal injection of a similar volume of $4 \%$ paraformaldehyde solution (SigmaAldrich) to preserve the pulmonary architecture. The inflated lungs were embedded in paraffin, stained with either H\&E, periodic acid Schiff(PAS), trichrome stain, or processed for immunohistochemistry. Lungs from the different experimental groups were all processed as a batch for either histologic staining or immunostaining under identical conditions. Stained and immunostained slides were all quantified under identical light microscope conditions, including magnification $(\times 20)$, gain, camera position, and background illumination. The quantitative histologic and image analysis of all coded slides was performed by research associates blinded to the coding of all the slides.

\section{BALF eosinophil counts}

Following instillation of $800 \mu$ l of sterile saline through the trachea into the lung, BALF was withdrawn, and cytospin preparations of BALF cells were made using a Shandon Cytospin centrifuge (Shandon Lipshaw Inc., Pittsburgh, Pennsylvania, USA). Eosinophil counts were performed as described previously (25).

\section{Quantitation of peribronchial fibrosis}

Three methods (trichrome stain, total lung collagen content, and peribronchial collagens III and V immunostaining) were used to quantify peribronchial fibrosis.

Peribronchial trichrome staining. The area of peribronchial trichrome staining in a paraffin-embedded lung was outlined and quantified using a light microscope (Leica DMLS; Leica Microsystems Inc., Depew, New York, USA) attached to an image-analysis system (Image-Pro Plus; Media Cybernetics, Silver Spring, Maryland, USA). Results are expressed as the area of trichrome staining per micrometer length of basement membrane of bronchioles with $150-200 \mu \mathrm{m}$ internal diameter. At least ten bronchioles were counted in each slide.

Lung collagen assay. The amount of lung collagen was measured using a collagen assay kit that uses a dye reagent that selectively binds to the $[\mathrm{Gly}-\mathrm{X}-\mathrm{Y}] n$ tripeptide sequence found within the triple helix sequence of mammalian collagens (Biocolor, Newtownabbey, Northern Ireland, United Kingdom), a method described for quantitation of lung collagen by Lee et al. (26). In all experiments a collagen standard was used to calibrate the assay.

Peribronchial collagens III and V immunostaining. For immunohistochemical detection of collagen III or collagen $V$, the lung sections were incubated with either a primary $\mathrm{mAb}$ (directed against mouse collagen III or V) (Polysciences Inc., Warrington, Pennsylvania, USA) or as a negative control mouse serum instead of the primary $\mathrm{Ab}$ for 30 minutes at room temperature. Immunoreactivity was detected by sequential incubations 
of lung sections with a biotinylated secondary $A b$, VECTASTAIN Rabbit ABC kit (Vector Laboratories, Burlingame, California, USA), followed by 3,3'-diaminobenzidine chromogen. The lung sections were briefly incubated with hematoxylin counterstain for 30 seconds and then mounted with aqueous mounting media. The area of peribronchial collagen III or V staining was outlined and quantified using a light microscope attached to an image-analysis system as described above for trichrome staining.

\section{Quantitation of peribronchial smooth muscle}

Airway smooth muscle thickness. The thickness of the airway smooth muscle layer was measured using an image-analysis system. Lungs that had been fixed in 3\% glutaraldehyde and $1 \%$ osmium tetroxide were stained with basic fuchsin-toluidine blue, which allowed the best visualization of the peribronchial smooth muscle layer. The thickness of the smooth muscle layer (the transverse diameter) was measured from innermost aspect to outermost aspect. The smooth muscle layer thickness was assessed at four predetermined bronchiole sites $\left(12,3,6\right.$, and $9 o^{\prime}$ 'clock) in at least ten bronchioles of similar size (150-200 $\mu \mathrm{m})$ on each slide.

$\alpha$-Smooth muscle actin immunostaining. Lung sections were processed in a similar manner for $\alpha$-smooth muscle actin immunostaining as described above for lung collagen immunostaining. The primary anti- $\alpha$-smooth muscle actin mAb was obtained from Sigma-Aldrich. The area of peribronchial $\alpha$-smooth muscle actin immunostaining was outlined and quantified using a light microscope attached to an image-analysis system as described above.

\section{Quantitation of TGF- $\beta$}

Levels of TGF- $\beta$ were quantitated by ELISA and by immunohistochemistry.

TGF- $\beta$ ELISA. The concentrations of TGF- $\beta 1$ (sensitivity of assay, $61 \mathrm{pg} / \mathrm{ml}$ ) were assayed in homogenized lung supernatants by ELISA (R\&D Systems Inc., Minneapolis, Minnesota, USA) using methods previously described in this laboratory (25). Prior to the TGF- $\beta 1$ assay, the BALF samples were treated with $2.5 \mathrm{~N}$ acetic acid to activate any latent TGF- $\beta 1(26,27)$.

TGF- $\beta$ immunobistochemistry. Serial sequential lung sections were processed for TGF- $\beta$ and MBP immunohistochemistry as described above for lung collagens III and $\mathrm{V}$ immunostaining, using an anti-TGF- $\beta \mathrm{Ab}$ (Santa Cruz Biotechnology Inc., Santa Cruz, California, USA) or an anti-mouse MBP Ab (James Lee, Mayo Clinic, Scottsdale, Arizona, USA). The number of individual cells staining positive for TGF- $\beta$ or MBP in the peribronchial space were counted using a light microscope. Results are expressed as the number of peribronchial cells staining positive for TGF- $\beta$ or MBP per bronchiole with $150-200 \mu \mathrm{m}$ of internal diameter. At least ten bronchioles were counted in each slide.

Cellular source of TGF- $\beta$. To characterize the peribronchial inflammatory cells contributing to TGF- $\beta$ expression, sequential thin lung cryosections $(3 \mu \mathrm{m})$ were immunostained by staining the first sequential lung section with an $\mathrm{Ab}$ to TGF- $\beta$ and the second sequential lung section with Ab's to either MBP (eosinophils), F4/80 (macrophages), or $\mathrm{CD} 4$ (CD4 $4^{+}$lymphocytes).

Airway epithelial $\alpha_{v} \beta_{6}$ integrin expression

Based on studies demonstrating that the integrin $\alpha_{v} \beta_{6}$ binds and activates latent TGF- $\beta$ and is expressed by stimulated airway epithelial cells $(28,29)$, we explored whether $\alpha_{v} \beta_{6}$ expression was induced in the remodeled airway epithelium by allergen challenge and could thus provide a mechanism for regulating latent TGF- $\beta$ activation in vivo. Lungs from WT and IL-5-deficient mice were immunostained with two different anti$\alpha_{v} \beta_{6}$ Ab's (mAb 2Q871; US Biological, Swampscott, Massachusetts, USA; mAb 10D5; Chemicon International, Temecula, California, USA), as well as speciesand isotype-matched Ab's, to determine levels of expression of $\alpha_{v} \beta_{6}$. The area of airway epithelial $\alpha_{v} \beta_{6}$ immunostaining was outlined and quantified using a light microscope attached to an image-analysis system as described above. Results are expressed as the area of $\alpha_{v} \beta_{6}$ immunostaining per micrometer length of basement membrane of bronchioles with 150-200 $\mu \mathrm{m}$ internal diameter. At least ten bronchioles were counted in each slide.

\section{Quantitation of airway mucus expression}

To quantitate the level of mucus expression in the airway, the number of PAS-positive and PAS-negative epithelial cells in individual bronchioles were counted as previously described in this laboratory (25). At least ten bronchioles were counted in each slide. Results are expressed as the percentage of PAS-positive cells per bronchiole, which is calculated from the number of PAS-positive epithelial cells per bronchus divided by the total number of epithelial cells of each bronchiole.

\section{Statistical analysis}

Results in the different groups of mice were compared by ANOVA using the nonparametric Kruskal-Wallis test followed by posttesting using Dunn's multiple comparison of means. All results are presented as mean plus or minus SEM. A statistical software package (Graph Pad Prism; GraphPad Software Inc., San Diego, California, USA) was used for the analysis. $P$ values of less than 0.05 were considered to be statistically significant.

\section{Results}

Repetitive OVA challenge induces BALF eosinophilia in WT but not IL-5-deficient mice

Three months of repetitive OVA challenge induced a significant BALF eosinophilia in WT mice compared with WT mice not challenged with OVA $\left(62.4 \pm 15.1 \times 10^{2}\right.$ versus $0.2 \pm 0.1 \times 10^{2}$ BALF eosinophils, WT OVA versus WT non-OVA challenged; $P<0.001$ ) (Figure 1). In contrast, the number of BALF eosinophils in repetitively 


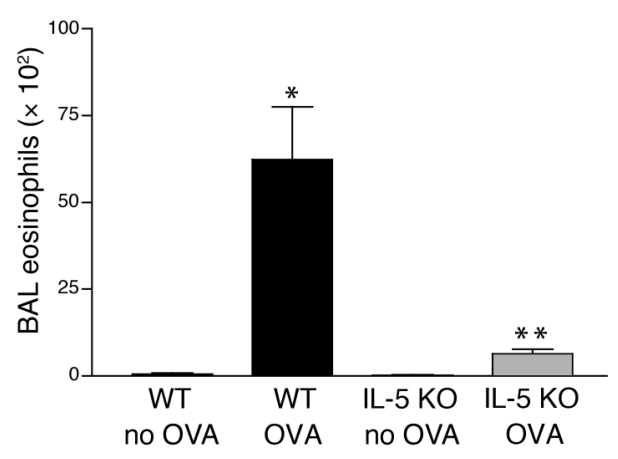

Figure 1

BALF eosinophil levels in WT and IL-5-deficient mice repetitively challenged with OVA. WT mice repetitively challenged with OVA for 3 months developed significantly increased numbers of BALF eosinophils compared with non-OVA-challenged WT mice ( ${ }^{*} \mathrm{WT}$ OVA versus WT no OVA; $P<0.001)$. In contrast IL-5-deficient mice repetitively challenged with OVA for 3 months had significantly reduced levels of BALF eosinophils compared with WT mice repetitively challenged with OVA for 3 months (**IL-5 KO OVA versus WT OVA; $P<0.01)$.

OVA-challenged IL-5-deficient mice were significantly reduced compared with WT OVA-challenged mice $(5.9 \pm$ $1.5 \times 10^{2}$ versus $62.4 \pm 15.1 \times 10^{2}$ BALF eosinophils, IL-5 KO OVA versus WT OVA; $P<0.01$ ) (Figure 1).

The number of peribronchial $\mathrm{CD}^{+}$lymphocytes $\left(13.1 \pm 1.5\right.$ versus $13.7 \pm 1.4 \mathrm{CD}^{+}$cells/bronchus, WT OVA versus IL-5-deficient OVA; $P=\mathrm{NS}$ ), as well as the number of $\mathrm{F} 4 / 80^{+}$macrophages $(36.7 \pm 3.8$ versus $30.2 \pm 2.5 \mathrm{~F} 4 / 80$ cells/bronchus, WT OVA versus IL-5deficient OVA; $P=\mathrm{NS}$ ), were not significantly different in IL-5-deficient versus WT mice repetitively challenged with OVA for 3 months. Repetitive OVA challenge induced a significant increase in BALF IL-5 in WT mice compared with IL-5-deficient mice (50.0 \pm 18.7 versus $<9.6 \mathrm{pg} / \mathrm{ml}$ BALF IL-5, WT OVA versus IL-5-deficient OVA; $P<0.05$ ), whereas there was no significant difference in the levels of another Th2 cytokine, IL-13, in the same groups of repetitively OVA-challenged mice $(489.2 \pm 35.2$ versus $486.1 \pm 19.4 \mathrm{pg} / \mathrm{ml}$ BALF IL-13, WT OVA versus IL-5-deficient OVA; $P=$ NS).

Repetitive OVA challenge induces less peribronchial fibrosis in IL-5-deficient versus WT mice

We used three different methods to quantitate peribronchial fibrosis in WT and IL-5-deficient mice, namely the area of peribronchial trichrome staining, total lung collagen content, and peribronchial III and $\mathrm{V}$ collagen immunostaining.

Repetitive OVA challenge in WT mice induced a significant increase in the area of peribronchial trichrome stain (Figure 2, $a$ and b) compared with non-OVAchallenged WT mice $(2.54 \pm 0.12$ versus $0.51 \pm 0.02$ $\mu \mathrm{m}^{2} / \mu \mathrm{m}$ circumference of bronchiole, WT OVA versus WT non-OVA challenged; $P<0.001$ ) (Figure 3a). In contrast, the area of trichrome staining in IL-5-deficient mice repetitively challenged with OVA (Figure 3a) was significantly reduced compared with WT mice repetitively challenged with OVA $(0.77 \pm 0.04$ versus $2.54 \pm 0.12 \mu \mathrm{m}^{2} / \mu \mathrm{m}$ circumference of bronchiole, IL-5 KO OVA versus WT OVA; $P<0.001$ ) (Figure $3 a$ ).

A single OVA challenge in WT mice did not induce airway remodeling as determined by either peribronchial trichrome staining or thickening of the peribronchial smooth muscle layer (data not shown). Comparison of peribronchial trichrome staining at different time points following the final 3-month OVA challenge in WT mice revealed that the peribronchial trichrome staining at 1-3 months after the last OVA challenge was approximately $75 \%$ of that noted 24 hours after the final 3-month OVA challenge (data not shown).

Repetitive OVA challenge in WT mice induced a significant increase in the levels of lung collagen compared with control non-OVA-challenged WT mice $(1,660 \pm 214$ versus $413 \pm 53 \mu \mathrm{g} /$ lung, WT OVA versus WT non-OVA challenged; $P<0.001$ ) (Figure $3 \mathrm{~b}$ ). In contrast, the levels of lung collagen in IL-5-deficient mice repetitively challenged with OVA were significantly reduced compared with WT mice repetitively challenged with OVA $(1,044 \pm 99$ versus $1,660 \pm 214 \mu \mathrm{g} / \mathrm{lung}, \mathrm{IL}-5 \mathrm{KO}$ OVA versus WT non-OVA challenged; $P<0.05$ ) (Figure $3 \mathrm{~b}$ ).

Repetitive OVA challenge in WT mice induced a significant increase in the area of peribronchial collagen III immunostaining compared with control non-OVAchallenged WT mice $(1.21 \pm 0.12$ versus $0.21 \pm 0.03$ $\mu \mathrm{m}^{2} / \mathrm{um}$ circumference of bronchiole, WT OVA versus WT non-OVA challenged; $P<0.001$ ) (Figure $3 c$ ), as well as collagen $\mathrm{V}$ immunostaining compared with control non-OVA-challenged WT mice $(1.35 \pm 0.11$ versus 0.16 $\pm 0.02 \mu \mathrm{m}^{2} / \mathrm{um}$ circumference of bronchiole, WT OVA
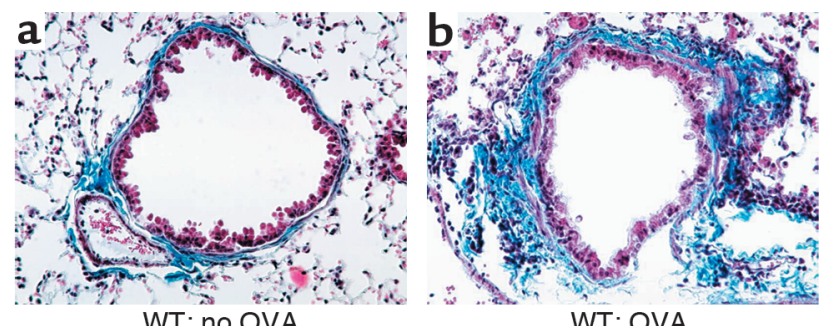

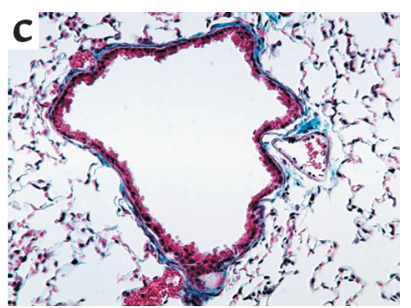

IL-5 KO: no OVA

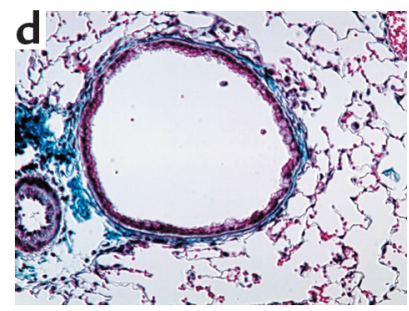

IL-5 KO: OVA

\section{Figure 2}

Peribronchial trichrome stain in WT and IL-5-deficient mice repetitively challenged with OVA. In the absence of OVA challenge, WT (a) and IL-5-deficient mice (c) exhibited minimal peribronchial trichrome staining (blue color). In contrast, repetitive OVA challenge in WT mice for 3 months induced circumferential peribronchial trichrome staining (b), which was significantly reduced in IL-5-deficient mice repetitively challenged with OVA $(\mathbf{d})$. 

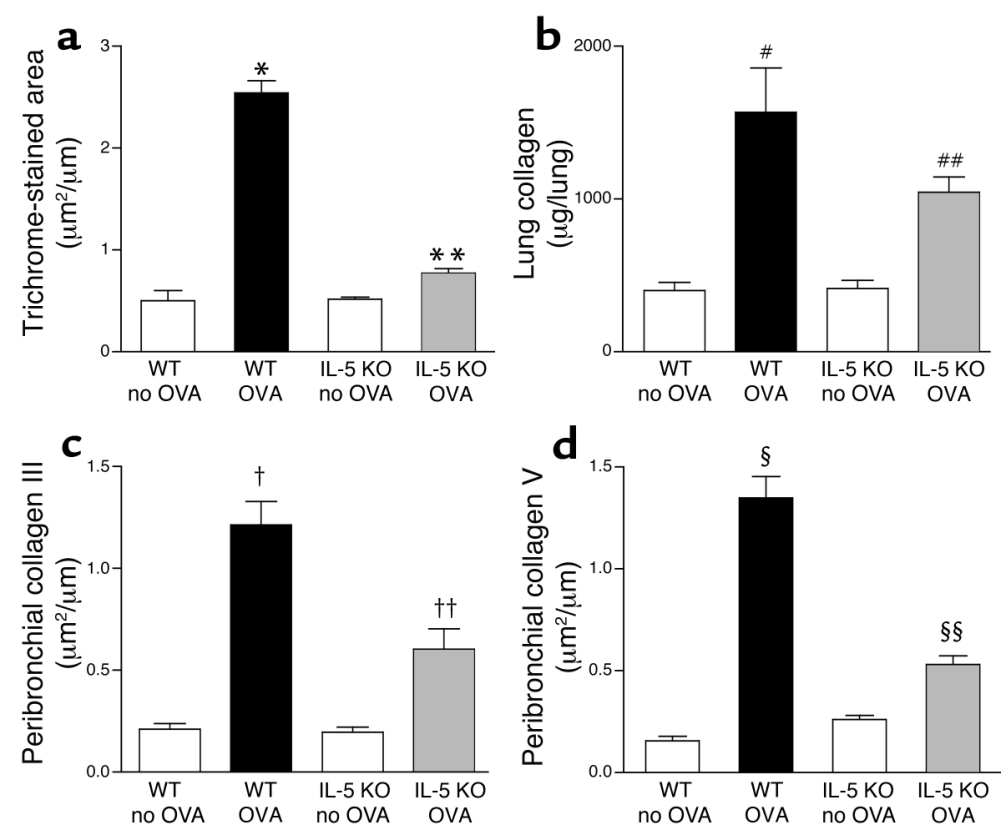

Figure 3

Quantitation of peribronchial fibrosis in WT and IL-5-deficient mice repetitively challenged with OVA. (a) Area of peribronchial trichrome stain. WT mice repetitively challenged with OVA for 3 months developed an increased area of peribronchial trichrome staining compared with non-OVA-challenged WT mice ( ${ }^{*}$ WT OVA versus WT no OVA; $P<0.001$ ). In contrast, IL-5-deficient mice repetitively challenged with OVA for 3 months had significantly reduced areas of peribronchial trichrome staining compared with WT mice repetitively challenged with OVA for 3 months (**IL-5 KO OVA versus WT OVA; $P<0.001)$. (b) Total lung collagen content. Repetitive OVA challenge induced a significant increase in total lung collagen in WT mice ("WT OVA versus WT no OVA; $P<0.001$ ). IL-5-deficient mice repetitively challenged with OVA had less total lung collagen compared with WT mice repetitively challenged with OVA (\#\#IL-5 KO OVA versus WT OVA; $P<0.05$ ). (c) Collagen III and (d) collagen V lung immunostaining. WT mice repetitively challenged with OVA for 3 months developed increased peribronchial collagen III immunostaining ( ${ }^{+W T}$ OVA versus WT no OVA; $\left.P<0.001\right)(\mathbf{c})$, as well as increased peribronchial collagen $V$ immunostaining compared with non-OVA-challenged WT mice (\$WT OVA versus WT no OVA; $P<0.001)(\mathbf{d})$. In contrast, IL-5-deficient mice repetitively challenged with OVA had significantly reduced levels of peribronchial collagen III immunostaining ( ${ }^{\dagger} I \mathrm{LL}-5$ KO OVA versus WT OVA; $P<0.001)(\mathbf{c})$, as well as significantly reduced levels of peribronchial collagen $V$ immunostaining, compared with WT mice challenged repetitively with OVA for 3 months ( $\$$ IL-5 KO OVA versus WT OVA; $P<0.001)($ d).

versus WT non-OVA challenged; $P<0.001$ ) (Figure 3d). In contrast, there was a significant reduction in the area of peribronchial collagen III immunostaining in IL-5-deficient mice repetitively challenged with OVA $\left(0.60 \pm 0.10\right.$ versus $1.21 \pm 0.12 \mu \mathrm{m}^{2} / \mathrm{um}$ circumference of bronchiole, IL-5 KO OVA versus WT OVA; $P<0.001$ ) (Figure 3c), as well as the area of peribronchial collagen $\mathrm{V}$ immunostaining in IL-5-deficient mice repetitively challenged with OVA compared with WT mice repetitively challenged with OVA $(0.53 \pm 0.04$ versus $1.35 \pm 0.11$ $\mu \mathrm{m}^{2} / \mathrm{um}$ circumference of bronchiole, IL-5 KO OVA versus WT OVA; $P<0.001$ ) (Figure $3 \mathrm{~d}$ ).

Repetitive OVA challenge induces less thickening of the peribronchial smooth muscle layer in IL-5-deficient versus WT mice

The thickness of the peribronchial smooth muscle layer (Figure 4a) in WT mice repetitively challenged with OVA was significantly greater than in control non-OVA-challenged WT mice $(11.8 \pm 0.4$ versus $3.4 \pm 0.1$ $\mu \mathrm{m}$, WT OVA versus WT non-OVA challenged; $P<0.001$ ) (Figure 4b). In contrast, the thickness of the peribronchial smooth muscle layer in IL-5-deficient mice repetitively challenged with OVA was significantly reduced compared with WT mice repetitively challenged with OVA $(5.6 \pm 0.3$ versus $11.8 \pm 0.4 \mu \mathrm{m}$, IL-5 KO OVA versus WT OVA; $P<0.001$ ) (Figure $4 \mathrm{~b}$ ).

The area of peribronchial $\alpha$-smooth muscle actin immunostaining in repetitively OVA-challenged WT mice was significantly greater than in control non-OVAchallenged WT mice $(1.28 \pm 0.06$ versus $0.34 \pm 0.02$ $\mu \mathrm{m}^{2} / \mu \mathrm{m}$ circumference of bronchiole, WT OVA versus WT non-OVA challenged; $P<0.001$ ) (Figure 4c). In contrast, the area of peribronchial $\alpha$-smooth muscle actin immunostaining in IL-5-deficient mice repetitively challenged with OVA was significantly reduced compared with WT mice repetitively challenged with OVA $\left(0.66 \pm 0.04\right.$ versus $1.28 \pm 0.06 \mu \mathrm{m}^{2} / \mu \mathrm{m}$ circumference of bronchiole, IL-5 KO OVA versus WT OVA; $P<0.001$ ) (Figure 4c).

Repetitive OVA challenge induces less lung TGF- $\beta$ expression in IL-5-deficient versus WT mice

Repetitive OVA challenge significantly increased the levels of lung TGF- $\beta$ in WT mice compared with control non-OVA-challenged WT mice (3,740 \pm 669 ver- 
a

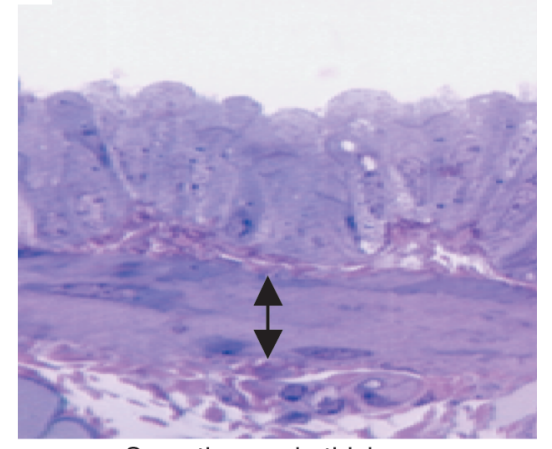

Smooth muscle thickness
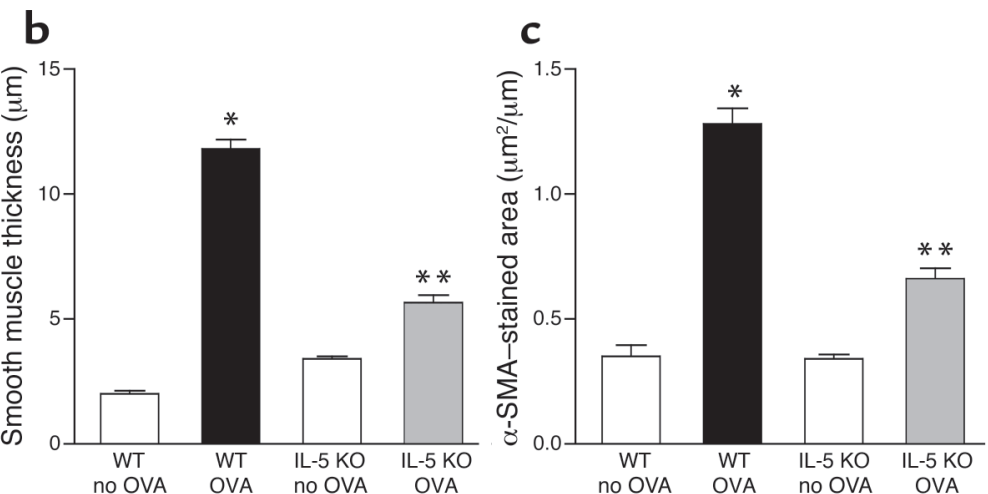

\begin{abstract}
Figure 4
Peribronchial smooth muscle layer in WT and IL-5-deficient mice repetitively challenged with OVA. (a) Peribronchial smooth muscle layer. Lungs that had been fixed in $3 \%$ glutaraldehyde and $1 \%$ osmium tetroxide were stained with basic fuchsin-toluidine blue, which allowed the best visualization of the peribronchial smooth muscle layer. The thickness of the smooth muscle layer (the transverse diameter) was measured from the innermost aspect to the outermost aspect of the smooth muscle layer (double arrow). (b) Peribronchial smooth muscle layer thickness. WT mice repetitively challenged with OVA for 3 months developed significantly increased thickness of the peribronchial smooth muscle layer compared with non-OVA-challenged WT mice ( ${ }^{*}$ WT OVA versus WT no OVA; $P<0.001$ ). In contrast, the thickness of the peribronchial smooth muscle layer in IL-5-deficient mice challenged repetitively with OVA was significantly reduced compared with WT mice challenged repetitively with OVA for 3 months ( ${ }^{*}$ IL-5 KO OVA versus WT OVA; $\left.P<0.001\right)$. (c) Peribronchial $\alpha$-smooth muscle actin $(\alpha-S M A)$. WT mice repetitively challenged with OVA for 3 months developed an increase in the area of peribronchial $\alpha$-smooth muscle actin immunostaining compared with non-OVA-challenged WT mice ( ${ }^{*}$ WT OVA versus WT no OVA; $\left.P<0.001\right)$. The area of peribronchial $\alpha$-smooth muscle actin immunostaining in IL-5-deficient mice challenged repetitively with OVA was significantly reduced compared with WT mice challenged repetitively with OVA for 3 months (**IL-5 KO OVA versus WT OVA; $P<0.001)$.
\end{abstract}

sus $1,480 \pm 456 \mathrm{pg} / \mathrm{ml}$, WT OVA versus WT non-OVA challenged; $P<0.01$ ) (Figure 5 a). Levels of lung TGF- $\beta$ were significantly reduced in IL-5-deficient mice repetitively challenged with OVA compared with WT mice repetitively challenged with OVA $(2,170 \pm 397$ versus $3,740 \pm 669 \mathrm{pg} / \mathrm{ml}$, IL-5 KO OVA versus WT OVA; $P<0.05)$ (Figure 5a).

Repetitive OVA-challenged IL-5-deficient mice have reduced numbers of MBP-positive and TGF- $\beta$-positive peribronchial cells

Repetitive OVA challenge in WT mice induced a significant increase in both the number of peribronchial MBP-positive cells ( $35.7 \pm 2.3$ versus $0.5 \pm 0.1 \mathrm{MBP}$-positive cells/bronchiole, WT OVA versus WT non-OVA challenged; $P<0.001$ ) (Figure 5, b and e), as well as a significant increase in the number of peribronchial TGF- $\beta$-positive cells ( $64.5 \pm 3.7$ versus $1.6 \pm 0.3$ TGF- $\beta$ positive cells/bronchiole, WT OVA versus WT non-OVA challenged; $P<0.001$ ) (Figure 5, $\mathrm{b}$ and $\mathrm{f}$ ) compared with non-OVA-challenged WT mice (Figure 5, c-d). In WT mice OVA challenge increased levels of epithelial cell TGF- $\beta$ immunostaining (Figure $5 \mathrm{f}$ ), as well as the number of peribronchial TGF- $\beta$-positive cells.

Repetitive OVA challenge in IL-5-deficient mice was associated with a parallel decrease in both the number of peribronchial MBP-positive cells $(2.2 \pm 0.5$ versus $35.7 \pm 2.3 \mathrm{MBP}$-positive cells/bronchiole, IL-5 KO OVA versus WT OVA; $P<0.001$ ) (Figure $5, \mathrm{~b}$ and $\mathrm{i}$ ), as well as a decrease in the number of peribronchial TGF- $\beta$-positive cells $(22.6 \pm 1.5$ versus $64.5 \pm 3.7$ TGF- $\beta$-positive cells/bronchiole, IL- 5 KO OVA versus
WT OVA; $P<0.001$ ) (Figure 5, b and j). Similar to experiments with WT mice, epithelial cell TGF- $\beta$ immunostaining increased in IL-5-deficient mice following OVA challenge (Figure $5 \mathrm{j}$ ).

To characterize the peribronchial inflammatory cells that contribute to TGF- $\beta$ expression, we used immunostaining to MBP (Figure 5k) and TGF- $\beta$ (Figure 5l) in sequential lung sections to demonstrate that eosinophils express TGF- $\beta$. Eosinophils (approximately $63 \%$ ) and F4/80-positive macrophages (approximately 35\%) were the major peribronchial inflammatory cellular sources of TGF- $\beta$ following repetitive OVA challenge for 3 months. We also noted significant TGF- $\beta$ expression by airway epithelium.

\section{Airway epithelial $\alpha_{v} \beta_{6}$ integrin expression}

Minimal airway epithelial $\alpha_{V} \beta_{6}$ expression can be detected in non-OVA-challenged WT (Figure 6a) or non-OVA-challenged IL-5-deficient mice (Figure 6b) in experiments using the anti- $\alpha_{v} \beta_{6}$ mAb 2Q871. Repetitive OVA challenge induced a significant increase in airway epithelial $\alpha_{v} \beta_{6}$ expression in WT mice $\left(0.46 \pm 0.09\right.$ versus $0.03 \pm 0.02 \mu \mathrm{m}^{2} / \mu \mathrm{m}$, WT OVA versus WT non-OVA challenged; $P<0.001$ ) (Figure $6, c$ and e). The area of airway epithelium staining positively for $\alpha_{v} \beta_{6}$ in repetitively OVA-challenged IL-5-deficient mice was significantly less than that of repetitively OVA-challenged WT mice $(0.24 \pm 0.06$ versus $0.46 \pm 0.09 \mu \mathrm{m}^{2} / \mu \mathrm{m}$, IL-5 KO OVA versus WT OVA; $P<0.001$ ) (Figure 6, $\mathrm{d}$ and e). Similar results were obtained using a different anti- $\alpha_{V} \beta_{6}$ mAb 10D5 (data not shown). 

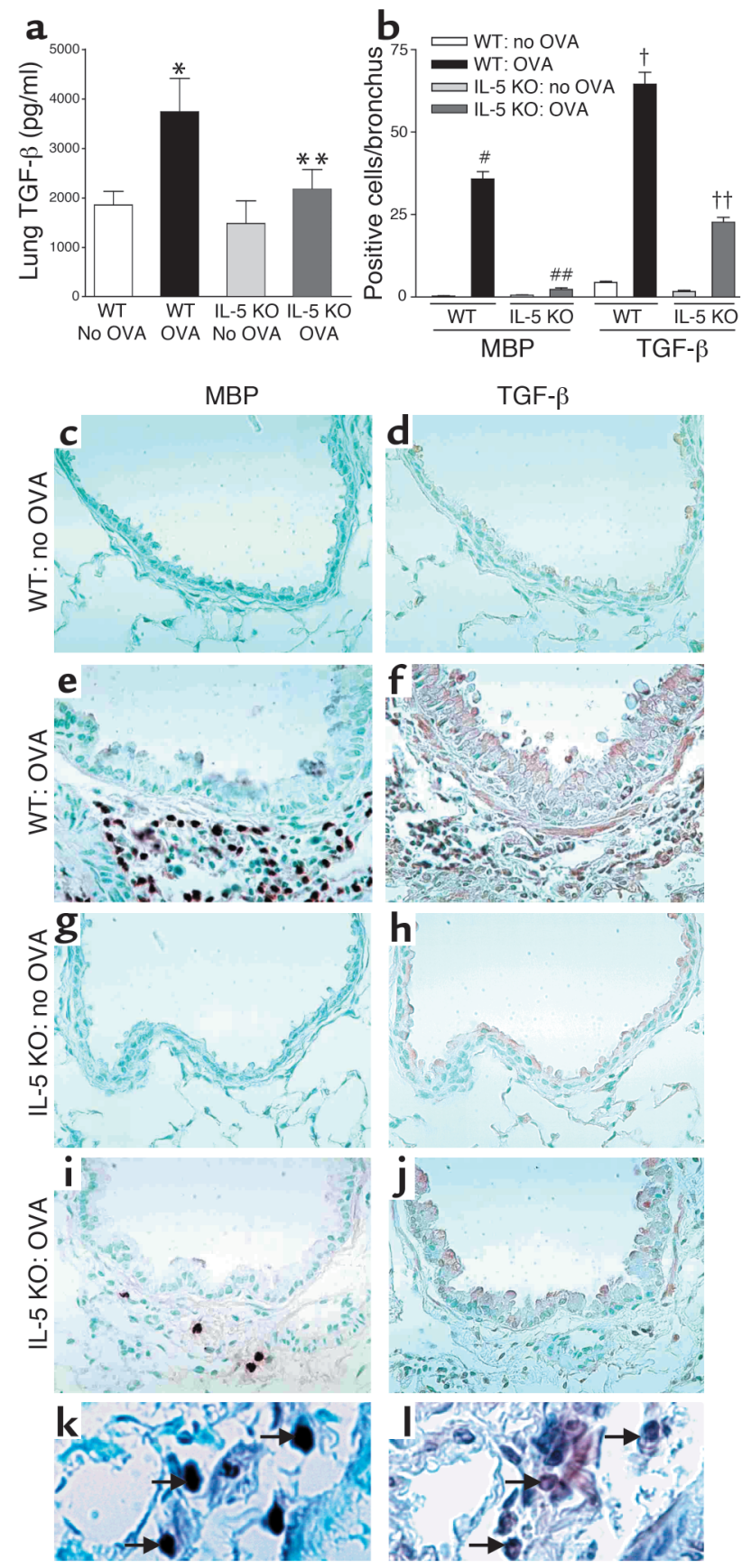

\section{Effect of IL-5 on airway mucus expression}

Repetitive OVA challenge in WT mice induced a significant increase in the percentage of airway epithelium that stained positive with PAS compared with non-OVA-challenged WT mice $(39.6 \% \pm 1.9 \%$ versus $0.5 \% \pm 0.2 \%$, WT OVA versus WT non-OVA challenged; $P<0.001)$. The percentage of airway epithelium staining positively with PAS in repetitively OVAchallenged IL-5-deficient mice was significantly less than that of repetitively OVA-challenged WT mice $(25.3 \% \pm 2.3 \%$ versus $39.6 \% \pm 1.9 \%$, IL-5 KO OVA versus WT OVA; $P<0.001)$.

\section{Figure 5}

Lung TGF- $\beta 1$ in WT and IL-5-deficient mice repetitively challenged with OVA. (a) WT mice challenged with OVA developed increased levels of lung TGF- $\beta 1$ compared with non-OVA-challenged WT mice $\left({ }^{*} \mathrm{WT}\right.$ OVA versus WT no OVA; $P<0.01$ ). Significantly reduced levels of TGF- $\beta 1$ were detected in IL-5-deficient mice versus WT mice challenged with OVA (**IL-5 KO OVA versus WT OVA; $P<0.05)$. (b) WT mice challenged with OVA had a significant increase in the number of peribronchial MBP-positive cells (\#WT OVA versus WT no OVA; $P<0.001$ ) and TGF- $\beta$-positive cells ( ${ }^{+W T}$ OVA versus WT no OVA; $P<0.001)$. There was a significant reduction in the number of MBP-positive cells (\#\#IL-5 KO OVA versus WT OVA; $P<0.001$ ) and TGF- $\beta$-positive cells ( ${ }^{+}$IL-5 KO OVA versus WT OVA; $P<0.001$ ) in IL-5-deficient mice challenged with OVA. (c-j) Non-OVA-challenged WT mice had few cells that immunostained positive for MBP (c) or TGF- $\beta$ (d). OVA-challenged WT mice had a significant increase in the number of cells that immunostain positive for $\operatorname{MBP}(\mathbf{e}) \operatorname{or} \mathrm{TGF}-\beta(\mathbf{f})$. OVA challenged IL-5-deficient mice had few cells that immunostained positive for MBP (i) or TGF- $\beta$ (j). Epithelial cells in OVA-challenged IL-5-deficient mice did immunostain positive for TGF- $\beta$ (j). Non-OVA-challenged IL-5-deficient mice had few cells that immunostained positive for $\mathrm{MBP}(\mathbf{g})$ or TGF- $\beta(\mathbf{h})$. The arrows in $\mathbf{k}$ point to three of four MBP-positive cells. The arrows in I point to three cells that are TGF- $\beta 1$ positive (corresponding to the same three MBP-positive cells in $\mathbf{k}$ ). Several additional TGF- $\beta 1$-positive cells that do not correspond to MBP-positive cells are also noted in $\mathbf{I}$.

\section{Discussion}

In this study we have used a novel model of allergeninduced airway remodeling to demonstrate that IL-5 plays an important role in airway remodeling as evidenced by reduction in two of the cardinal structural features associated with airway remodeling in asthma, namely peribronchial fibrosis and thickening of the peribronchial smooth muscle layer (17-19). The reduced peribronchial fibrosis in IL-5-deficient mice was evident from studies measuring lung collagen content, immunohistochemical staining of airways for collagen III and V deposition, and peribronchial trichrome staining. In humans, the basement membrane of airway epithelium comprises two layers, the basal lamina (referred to as the true basement membrane, of normal thickness in asthmatic airways) and the lamina reticularis (composed of collagen types I, III, V, and fibronectin), which is thickened in asthmatic airways (17). Immunohistochemical staining of the airways of WT mice repetitively challenged with OVA demonstrated increased peribronchial deposition of collagens III and $\mathrm{V}$, collagen types noted in the remodeled airway of human asthmatics (17). The repetitive OVA challenge in WT mice was not only associated with peribronchial fibrosis, but also with increased peribronchial smooth muscle thickness. Both peribronchial fibrosis and the thickness of the peribronchial smooth muscle layer were significantly reduced in IL-5-deficient compared with WT mice. Although IL-5-deficient mice had significantly reduced levels of airway remodeling, airway remodeling changes were not completely ablated in IL-5-deficient mice, suggesting that additional cytokines/mediators other than IL-5 are also involved in allergen-induced airway remodeling. 
The airways of repetitively OVA-challenged IL-5-deficient mice had significantly less eosinophils compared with OVA challenged WT mice. This reduction in peribronchial eosinophil accumulation was associated with a significant reduction in the number of peribronchial cells expressing TGF- $\beta$ as assessed by immunohistochemistry, as well as by a significant reduction in total lung TGF- $\beta$. Because eosinophils are known to express TGF- $\beta$ (20-23), the parallel reduction in the number of peribronchial eosinophils and the number of peribronchial cells expressing TGF- $\beta$ in IL-5-deficient mice suggests that eosinophils may be a significant source of TGF- $\beta$ in the remodeled airway in mice repetitively challenged with OVA. Combination immunostaining of sequential lung sections with Ab's to MBP and TGF- $\beta$ confirmed that eosinophils were a significant source of TGF- $\beta$ in the remodeled airway. TGF- $\beta$ expression in the remodeled airway of WT mice could account for many of the features of airway remodeling noted. Because TGF- $\beta$ stimulates fibroblasts to produce ECM proteins such as collagen (27), the increased levels of TGF- $\beta$ expression detected in WT mice challenged with OVA could contribute to fibroblast collagen synthesis, whereas the reduction in TGF- $\beta$ expression could account for the reduced collagen synthesis noted in OVA-challenged IL-5-deficient mice.

The TGF- $\beta 1$ gene encodes a procytokine consisting of a C-terminal TGF- $\beta 1$ sequence and a larger $\mathrm{N}$-terminal region that, after processing, forms a protein called latency-associated peptide (LAP) $(27,28)$. LAP and TGF- $\beta 1$ remain noncovalently associated and in this configuration TGF- $\beta 1$ is latent as LAP blocks binding of TGF- $\beta 1$ to its receptor. Previous studies have demonstrated that the integrin $\alpha_{V} \beta_{6}$ binds and activates latent TGF- $\beta 1$ (28) and that $\alpha_{V} \beta_{6}$ has a very restricted pattern of tissue expression being only expressed in epithelium, particularly lung and skin epithelium $(29,30)$. In addition, $\alpha_{v} \beta_{6}$ is not expressed in unstimulated airway epithelium, but can be induced in vivo by stimuli such as epithelial injury (e.g., intravenous bacterial infection that causes acute lung injury in mice) or epithelial repair (30). Because the epithelialexpressed integrin $\alpha_{v} \beta_{6}$ binds and activates latent TGF- $\beta 1(28,29)$, we explored whether airway remodeling in WT mice was associated with increased levels of epithelial $\alpha_{v} \beta_{6}$ integrin expression. To our knowledge, no previous studies have demonstrated that airway remodeling in asthma is associated with expression of epithelial $\alpha_{v} \beta_{6}$ integrin or that there is a link between eosinophils and $\alpha_{v} \beta_{6}$ integrin expression. We used two different $A b$ 's (which detect different epitopes on the combined heterodimer $\alpha_{V} \beta_{6}$ integrin) to demonstrate by immunohistochemistry that repetitive OVA challenge for 3 months induces a significant increase in levels of airway epithelial $\alpha_{v} \beta_{6}$ expression. In contrast, diluent-challenged control mice express minimal levels of airway epithelial $\alpha_{v} \beta_{6}$. Interestingly, levels of expression of airway epithelial $\alpha_{v} \beta_{6}$ were significantly reduced in the lungs of repetitively OVA-challenged IL-5-defi- cient mice compared with OVA-challenged WT mice, suggesting that IL-5 and/or eosinophils were inducing epithelium to express $\alpha_{v} \beta_{6}$. Because IL-5 receptors are present on eosinophils rather than epithelium, it is unlikely that IL-5 is directly inducing airway epithelial cells to express $\alpha_{v} \beta_{6}$, but more likely that TGF- $\beta$, or another mediator derived from eosinophils, is upregulating $\alpha_{v} \beta_{6}$ expression by epithelium. Because TGF- $\beta$ upregulates expression of $\beta 6$ integrins in cultured airway epithelial cells in vitro (30), expression of TGF- $\beta$ by eosinophils and other cells in the remodeled airway could account for the upregulation of $\beta 6$ integrin expression in epithelium that subsequently activates

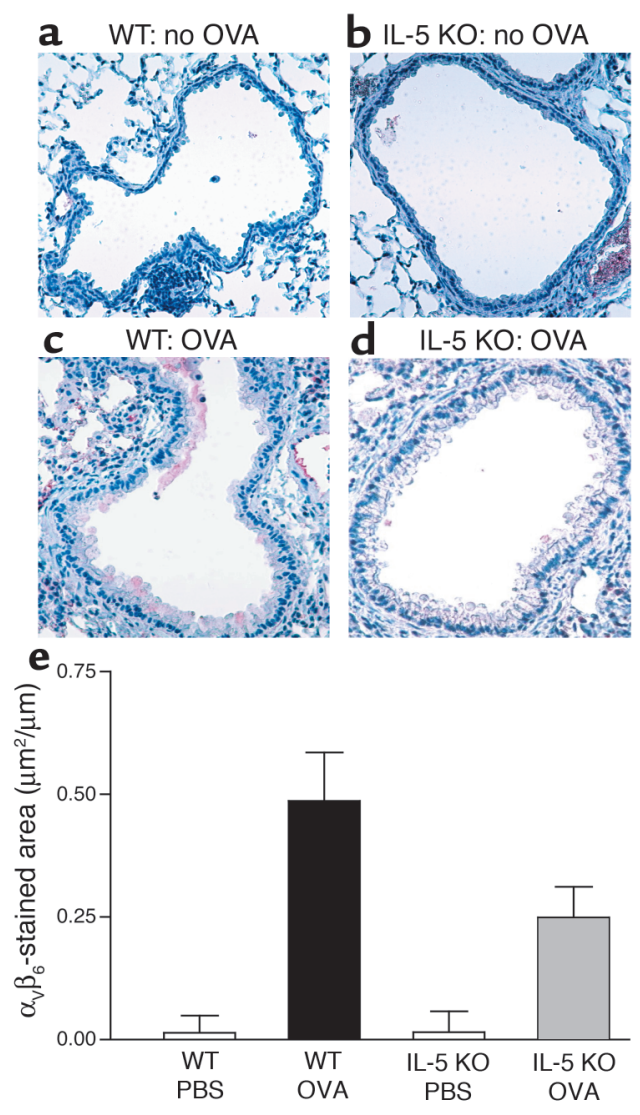

\section{Figure 6}

Expression of airway epithelial integrin $\alpha_{v} \beta_{6}$ in WT and IL-5 KO mice. Neither non-OVA-challenged WT mice (a) nor non-OVA-challenged IL-5-deficient mice (b) had significant numbers of airway epithelial cells that immunostained positive for $\alpha_{V} \beta_{6}$. In contrast, WT mice challenged repetitively with OVA for 3 months had a significant increase in the number of airway epithelial cells that immunostained positive for $\alpha_{v} \beta_{6}(\mathbf{c})$. There was a significant reduction in IL-5-deficient mice in the number of airway epithelial cells that immunostain positive for $\alpha_{v} \beta_{6}$ following repetitive OVA challenge (d). Quantitative analysis of $\alpha_{v} \beta_{6}$ integrin expression in airway epithelial cells demonstrated that repetitive OVA challenge in WT mice induced a significant increase in $\alpha_{V} \beta_{6}$ expression in WT mice compared with diluent-challenged WT mice (WT OVA versus WT no OVA; $P<0.001$ ). The area of airway epithelium staining positively for $\alpha_{v} \beta_{6}$ in repetitively OVA-challenged IL-5-deficient mice was significantly less than that of repetitively OVA-challenged WT mice (IL-5 KO OVA versus WT OVA) $(P<0.001)(\mathbf{e})$. 
latent TGF- $\beta$ to its bioactive form. Thus, a consequence of reduced levels of eosinophils in IL-5-deficient mice may include reduced epithelial expression of $\alpha_{v} \beta_{6}$, with reduced activation of latent peribronchial TGF- $\beta$ to bioactive TGF- $\beta$, as well as reduced levels of fibrosis.

IL-5-deficient mice repetitively challenged with OVA had less remodeling of their airways, and this was associated with less epithelial mucus expression compared with WT mice. The mechanism by which IL-5 and/or eosinophils induce epithelial mucus expression is not completely understood. The importance of eosinophils to mucus expression has been demonstrated in studies in which eosinophils were transferred directly into the lungs of acute OVA-challenged IL-5-deficient mice (9). In these studies eosinophil transfer restored epithelial mucus expression and airway hyperresponsiveness in IL-5-deficient mice to levels similar to that noted in acute OVA-challenged WT mice (9). Further studies suggesting a direct role for IL-5 and eosinophils in inducing mucus expression include studies with IL-5-transgenic mice that constitutively express IL-5 in the lung epithelium (5). These epithelial IL-5-transgenic mice develop accumulation of peribronchial eosinophils that is associated with goblet cell hyperplasia and airway hyperresponsiveness to methacholine in the absence of aerosolized antigen challenge (5).

Previous studies have also investigated a potential role for IL-5 in mouse models of airway remodeling, using different methods of inducing and assessing it $(31,32)$. Blyth et al. (31) administered three intratracheal instillations of OVA antigen over a 10-day period to mice sensitized to OVA and demonstrated a 1.4fold increase in subepithelial reticulin using Gordon and Sweets stain for identification of reticulin. The reticulin staining was reduced in OVA-challenged mice by pretreatment with an anti-IL-5 Ab (31). Foster et al. (32) studied the effect of aerosol delivery of OVA to sensitized mice on inflammatory and tracheal epithelial responses and demonstrated a dissociation of inflammatory and tracheal epithelial responses in a murine model (32). The model of airway remodeling we have developed induces sustained expression in WT mice of Th2 cytokines including IL-5, IL-9, and IL-13 (24) and a robust remodeling response in the lung, which has allowed us to investigate features and mechanisms of allergen-induced remodeling that have not been investigated previously in other models. Some of the features of airway fibrosis we have identified in this allergen-induced model of airway remodeling have been observed in in vivo studies with transgenic mice that overexpress IL-13 in airway epithelium (26). The epithelial-targeted IL-13-transgenic mice exhibit lung fibrosis that is significantly reduced by treatment with a TGF- $\beta 1$ antagonist, suggesting an important role for TGF- $\beta 1$ in mediating the fibrotic effects of IL-13 in this model (26). Interestingly, recent studies provide evidence that IL-5 can modulate IL-13 production from Th2 cells, suggest- ing that these two cytokine signaling systems are not necessarily independent effector pathways and may also be integrated to regulate aspects of the allergic inflammatory response (10).

In vivo studies in atopic subjects have also suggested a role for eosinophils and TGF- $\beta$ in tissue remodeling in humans as opposed to the aforementioned studies in mice (33). Skin biopsies derived from allergeninduced cutaneous late-phase reactions have demonstrated that expression of TGF- $\beta$ by eosinophils at 6 hours after challenge is associated with myofibroblast formation and deposition of tenascin and procollagen I at 24-48 hours after challenge (33). In vitro coculture of eosinophils with fibroblasts induces fibroblasts to express $\alpha$-smooth muscle actin and tenascin (33). $A b$ 's to TGF- $\beta$ inhibit fibroblasts cocultured with eosinophils from expressing $\alpha$-smooth muscle actin (33). Recent studies have demonstrated that mild atopic asthmatics treated with anti-IL-5 for 2 months have reduced numbers of eosinophils in bronchial mucosa as well as reduced expression of tenascin, lumican, and procollagen III in the bronchial mucosal reticular basement membrane when compared with placebo (34). In addition, anti-IL-5 treatment was associated with a significant reduction in the numbers and percentage of airway eosinophils expressing mRNA for TGF- $\beta 1$ and the concentration of TGF- $\beta 1$ in BALF (34). At present, there are no results from human studies as to whether anti-IL-5 influences smooth muscle layer thickness or levels of expression of airway epithelial $\alpha_{V} \beta_{6}$ integrin expression.

In summary, in this study we have demonstrated an important role for IL-5 in airway remodeling. This conclusion is supported by the observation that IL-5deficient mice had significantly less peribronchial fibrosis (total lung collagen content, peribronchial collagens III and V), and significantly less peribronchial smooth muscle (thickness of the peribronchial smooth muscle layer, $\alpha$-smooth muscle actin) compared with WT mice. Repetitively OVAchallenged IL-5-deficient mice had a significant reduction in the number of peribronchial cells staining positive for MBP, which was paralleled by a similar reduction in the number of cells staining positive for TGF- $\beta$, suggesting that IL-5, eosinophils, and TGF- $\beta$ play an important role in airway remodeling. OVA challenge also induced significantly higher levels of airway epithelial $\alpha_{v} \beta_{6}$ integrin expression and bioactive lung TGF- $\beta$ in WT compared with IL-5deficient mice. Increased airway expression of $\alpha_{V} \beta_{6}$ may thus contribute to increased activation of latent TGF- $\beta$. These results suggest an important role for IL-5, eosinophils, $\alpha_{V} \beta_{6}$, and TGF- $\beta$ in airway remodeling. Because initial human studies with anti-IL-5 have not noted improvement in airway hyperreactivity in mild asthmatics, further studies of the effect of anti-IL-5 on parameters of airway remodeling will assist in assessing the potential value of anti-IL-5 therapy in asthma. 


\section{Acknowledgments}

We acknowledge the contributions to these studies of Manfred Kopf (Swiss Federal Institute of Technology, Zurich, Switzerland), who kindly provided the IL-5deficient mice, and James Lee (Mayo Clinic, Scottsdale, Arizona, USA), who kindly provided the anti-mouse MBP Ab. This study was supported by NIH grants AI33977 and AI-38425 (to David Broide).

1. Rothenberg, M.E. 1998. Eosinophilia. N. Engl. J. Med. 338:1592-1600. 2. Broide, D. 2002. Fast flowing eosinophils: signals for stopping and stepping out of blood vessels. Am. J. Respir. Cell Mol. Biol. 26:637-640.

3. Lopez, A.F., et al. 1988. Recombinant human interleukin 5 is a selective activator of human eosinophil function. J. Exp. Med. 167:219-224.

4. Foster, P.S., Hogan, S.P., Ramsay, A.J., Matthaei, K.I., and Young, I.G. 1996. Interleukin 5 deficiency abolishes eosinophilia, airways hyperreactivity, and lung damage in a mouse asthma model. J. Exp. Med. 183:195-201.

5. Lee, J.J., et al. 1997. Interleukin-5 expression in the lung epithelium of transgenic mice leads to pulmonary changes pathognomonic of asthma. J. Exp. Med. 185:2143-2156.

6. Hamelmann, E., et al. 1997. Anti-interleukin-5 antibody prevents airway hyperresponsiveness in a murine model of airway sensitization. Am.J. Respir. Crit. Care Med. 155:819-825.

7. Hamelmann, E., et al. 1999. Anti-interleukin 5 but not anti-IgE prevent airway inflammation and airway hyperresponsiveness. Am. J. Respir. Crit. Care Med. 160:934-941.

8. Corry, D, et al. 1996. Interleukin 4, but not interleukin 5 or eosinophils, is required in a murine model of acute airway hyperreactivity. J. Exp. Med. 183:109-117.

9. Shen, H.H., et al. 2003. A causative relationship exists between eosinophils and the development of allergic pulmonary pathologies in the mouse. J. Immunol. 170:3296-3305.

10. Mattes, J., et al. 2002. Intrinsic defect in T cell production of interleukin (IL)-13 in the absence of both IL-5 and eotaxin precludes the development of eosinophilia and airways hyperreactivity in experimental asthma. J. Exp. Med. 195:1433-1444.

11. Robinson, D.S., et al. 1992. Predominant TH2-like bronchoalveolar Tlymphocyte population in atopic asthma. N. Engl. J. Med. 326:298-304

12. Broide, D.H., Paine, M.M., and Firestein, G.S. 1992. Eosinophils express interleukin 5 and granulocyte macrophage-colony-stimulating factor mRNA at sites of allergic inflammation in asthmatics. J. Clin. Invest. 90:1414-1424.

13. Sur, S., Gleich, G.J., Swanson, M.C., Bartemes, K.R., and Broide, D.H. 1995. Eosinophilic inflammation is associated with elevation of interleukin-5 in the airways of patients with spontaneous symptomatic asthma. J. Allergy Clin. Immunol. 96:661-668.

14. Shi, H.Z., et al. 1998. Effect of inhaled interleukin-5 on airway hyperreactivity and eosinophilia in asthmatics. Am. J. Respir. Crit. Care Med. 157:204-209.

15. Leckie, M.J., et al. 2000. Effects of an interleukin-5 blocking monoclonal antibody on eosinophils, airway hyper-responsiveness, and the late asthmatic response. Lancet. 356:2144-2148.
16. Flood-Page, P.T., Menzies-Gow, A.N., Kay, A.B., and Robinson, D.S. 2003. Eosinophil's role remains uncertain as anti-interleukin- 5 only partially depletes numbers in asthmatic airway. Am. J. Respir. Crit. Care Med. 167:199-204.

17. Elias, J.A., Zhu, Z., Chupp, G., and Homer, R.J. 1999. Airway remodeling in asthma. J. Clin. Invest. 104:1001-1006.

18. Roche, W.R., Beasley, R., Williams, J., and Holgate, S.T. 1989. Subepithelial fibrosis in the bronchi of asthmatics. Lancet. 1:520-524.

19. Johnson, P.R., et al. 2001. Airway smooth muscle cell proliferation is increased in asthma. Am. J. Resp. Crit. Care. Med. 164:474-477.

20. Ohno, I., et al. 1996. Transforming growth factor beta 1 (TGF beta 1) gene expression by eosinophils in asthmatic airway inflammation. Am. J. Respir. Cell Mol. Biol. 15:404-409.

21. Minshall, E.M., et al. 1997. Eosinophil-associated TGF-beta1 mRNA expression and airways fibrosis in bronchial asthma. Am. J. Respir. Cell Mol. Biol. 7:326-333.

22. Elovic, A.E., et al. 1998. IL-4-dependent regulation of TGF-alpha and TGF-beta 1 expression in human eosinophils. J. Immunol. 160:6121-6127.

23. Ohkawara, Y., et al. 2000. Activation and transforming growth factorbeta production in eosinophils by hyaluronan. Am. J. Respir. Cell Mol. Biol. 23:444-451.

24. Ikeda, R.K., et al. 2003. Accumulation of peribronchial mast cells in a mouse model of ovalbumin allergen induced chronic airway inflammation: modulation by immunostimulatory DNA sequences. J. Immunol. 171:4860-4867.

25. Ikeda, R.K., et al. 2003. Resolution of airway inflammation following OVA inhalation: comparison of ISS DNA and corticosteroids. Am. J. Resp. Cell Mol. Biol. 28:655-663.

26. Lee, C.G., et al. 2001. Interleukin-13 induces tissue fibrosis by selectively stimulating and activating transforming growth factor beta(1). J. Exp. Med. 194:809-821.

27. Blobe, G.C., Schiemann, W.P., and Lodish, H.F. 2000. Role of transforming growth factor beta in human disease. New. Engl. J. Med. 342:1350-1358.

28. Munger, J.S., et al. 1999. The integrin $\alpha V \beta 6$ binds and activates latent TGF- $\beta 1$ : a mechanism for regulating pulmonary inflammation and fibrosis. Cell. 96:319-328.

29. Huang, X.Z., et al. 1996. Inactivation of the integrin beta 6 subunit gene reveals a role of epithelial integrins in regulating inflammation in the lung and skin. J. Cell Biol. 133:921-928

30. Breuss, J.M., et al. 1995. Expression of the $\beta 6$ integrin subunit in development, neoplasia, and tissue repair suggests a role in epithelial remodeling. J. Cell Sci. 108:2241-2251.

31. Blyth, D.I., Wharton, T.F., Pedrick, M.S., Savage, T.J., and Sanjar, S. 2000. Airway subepithelial fibrosis in a murine model of atopic asthma: suppression by dexamethasone or anti-interleukin-5 antibody. Am. J. Respir. Cell Mol. Biol. 23:241-246.

32. Foster, P.S., et al. 2000. Dissociation of inflammatory and epithelial responses in a murine model of chronic asthma. Lab. Invest. 80:655-662

33. Phipps, S., et al. 2002. The relationship between allergen-induced tissue eosinophilia and markers of repair and remodeling in human atopic skin. J. Immunol. 169:4604-4612.

34. Flood-Page, P., et al. 2003. Anti-IL-5 treatment reduces deposition of ECM proteins in the bronchial subepithelial basement membrane of mild atopic asthmatics. J. Clin. Invest. 112:1029-1036. doi:10.1172/JCI200317974. 